\title{
LOCAL RESTRICTIONS FOR VARIOUS CLASSES OF DIRECTED GRAPHS
}

\section{W. BeIneke and F. Harary}

The purpose of this article is to provide and establish criteria for determining whether a sequence of ordered pairs of integers is the sequence of degrees of the points of a directed graph possessing particular properties. After some necessary definitions and a preliminary result, such criteria are given for digraphs with different kinds of connectedness. In the final two sections we consider digraphs having the additional properties of being functional and traversable.

\section{Preliminaries}

A directed graph, or more briefly a digraph $D$, consists of a finite nonempty set $V=\left\{v_{1}, v_{2}, \ldots, v_{p}\right\}$ of points together with a given subset $X$ of $V \times V$. The elements of $X$ are called lines, and for any line $x=v_{i} v_{j}=\left(v_{i}, v_{j}\right)$, it is specified that $v_{i} \neq v_{j}$. Also, $v_{i}$ is then said to be adjacent to $v_{j}$ and $v_{j}$ adjacent from $v_{i}$. The outdegree of a point $v_{i}$, denoted by od $v_{i}=a_{i}$, is the number of points adjacent from $v_{i}$, and its indegree, $\operatorname{id} v_{i}=b_{i}$, is the number of points adjacent to it. The degree of point $v_{i}$ is then the ordered pair $\left(a_{i}, b_{i}\right)$, and the degree sequence $\sigma(D)$ of the digraph $D$ is the sequence of degrees of its points, that is,

$$
\sigma(D)=\left(\left(a_{1}, b_{1}\right),\left(a_{2}, b_{2}\right), \ldots,\left(a_{p}, b_{p}\right)\right) .
$$

A sequence of points and lines of the form $u_{1}, u_{1} u_{2}, u_{2}, u_{2} u_{3}, \ldots, u_{n-1} u_{n}$, $u_{n}$, in which the $n$ points are distinct, is called a path from $u_{1}$ to $u_{n}$. If there is a path from $u$ to $v$ in $D$, then $v$ is said to be reachable from $u$. A semipath joining $u$ and $v$ is a sequence of points and lines which would become a path if the direction of some (possibly none) of the lines were reversed.

A digraph $D$ is strongly connected, or strong, if every pair of points are mutually reachable; $D$ is weakly connected, or weak, if every pair of points are joined by a semipath. A digraph is disconnected if it is not even weak.

A subgraph of $D$ consists of subsets of the points and lines of $D$ which themselves constitute a digraph. A strong component $S$ of a digraph $D$ is a maximal strongly connected subgraph; similarly a weak component $W$ of $D$ is a maximal weakly connected subgraph.

The trivial digraph consists of a single point, as does a trivial strong component of a digraph. A trivial weak component is called an isolated point.

Received 15 July, 1963. The preparation of this article was supported by the National Science Foundation, U.S.A., under grant GP-207. 
For any line $x$ of $D$, let $D-x$ be the maximal subgraph not containing $x$. If $u$ and $v$ are distinct points of $D$ and line $y=u v$ is not in $D$, call $D+y$ the union of $D$ with line $y$.

Let $\sigma$ be a sequence of $p$ ordered pairs of non-negative integers. Fulkerson [2] and Ryser [6] have found the criteria of Theorem 0 for $\sigma$ to be the degree sequence of some digraph. If there is such a digraph $D$, we say that $\sigma$ is graphical, that $\sigma$ belongs to $D$, and that $D$ is a digraph of $\sigma$.

Theorem 0 . Let $\sigma$ be a sequence of ordered pairs of non-negative integers $\left(\left(a_{1}, b_{1}\right),\left(a_{2}, b_{2}\right), \ldots,\left(a_{p}, b_{p}\right)\right)$, in which $a_{1} \geqslant a_{2} \geqslant \ldots \geqslant a_{p}$, $0 \leqslant a_{i} \leqslant p-1$, and $0 \leqslant b_{i} \leqslant p-1$. This sequence is graphical if and only if the equation

$$
\sum_{i=1}^{p} a_{i}=\sum_{i=1}^{p} b_{i}
$$

and the following $p-1$ inequalities hold:

$$
\sum_{i=1}^{k} a_{i} \leqslant \sum_{i=1}^{k} \min \left\{k-1, b_{i}\right\}+\sum_{i=k+1}^{p} \min \left\{k, b_{i}\right\} ; \quad 1 \leqslant k \leqslant p-1 .
$$

\section{Degree sequences and kinds of connectedness}

We will develop necessary and sufficient conditions for a graphical sequence $\sigma$ to belong to disconnected, weak, and strong digraphs. The trivial digraph, whose degree sequence is $((0,0))$, is both weak and strong. Hence we only consider nontrivial digraphs.

Theorem 1. A graphical sequence $\sigma=\left(\left(a_{1}, b_{1}\right),\left(a_{2}, b_{2}\right), \ldots,\left(a_{p}, b_{p}\right)\right)$ belongs to some disconnected digraph if and only if $\sigma$ can be partitioned into two graphical subsequences.

Both the necessity and sufficiency of this condition are immediate and their proofs are omitted. Instead we illustrate this with

$$
\sigma=((2,2),(2,2),(2,1),(1,2),(1,1),(1,1)) .
$$

This sequence is graphical and partitions into the two graphical subsequences

$$
((2,2),(2,1),(1,2)) \text { and }((2,2),(1,1),(1,1)) \text {. }
$$

Theorem 2. A graphical sequence $\sigma=\left(\left(a_{1}, b_{1}\right),\left(a_{2}, b_{2}\right), \ldots,\left(a_{p}, b_{p}\right)\right)$ belongs to some weak digraph if and only if it does not contain $(0,0)$ and

$$
\sum_{i=1}^{p} a_{i} \geqslant p-1
$$


Proof. If $\sigma$ is the degree sequence of a weak digraph $D$ with $p$ points, then $\sigma$ does not contain $(0,0)$ since $D$ has no isolated points. Of course $D$ has at least $p-1$ lines, as mentioned in [1]. Therefore, the necessity of these conditions is immediate.

For the sufficiency, suppose $\sigma$ is a sequence which satisfies these conditions but belongs to no weak digraph. Let $D$ be a digraph of $\sigma$ with the minimum number of weak components. Then it can easily be shown that some weak component $W$ has a line $x=u v$ such that $W-x$ is also weakly connected. Let $x^{\prime}=u^{\prime} v^{\prime}$ be a line in any other weak component $W^{\prime}$. Define two new lines $y_{1}=u v^{\prime}$ and $y_{2}=u^{\prime} v$. Then $E=D-x-x^{\prime}+y_{1}+y_{2}$ is also a digraph of $\sigma$, and we now show that $E$ has fewer weak components than $D$. Let $w$ and $w^{\prime}$ be arbitrary points of $W$ and $W^{\prime}$ respectively. In $W-x$ there are semipaths $P$ and $Q$ joining $w$ with $u$ and $v$ respectively. In $W^{\prime}-x^{\prime}$ there is a semipath $P^{\prime}$ joining $u^{\prime}$ and $w^{\prime}$ or a semipath $Q^{\prime}$ joining $v^{\prime}$ and $w^{\prime}$. If $P^{\prime}$ exists, then $P^{\prime}+y_{2}+Q$ is a semipath joining $w^{\prime}$ and $w$; otherwise $P+y_{1}+Q^{\prime}$ is such a semipath. Therefore any two points of $W \cup W^{\prime}$ are joined by a semipath in $E$. Hence, $E$ has fewer weak components than $D$, contrary to the supposition.

In order to develop a criterion for a sequence $\sigma$ to belong to a strong digraph, we require the following preliminary observation.

Lemma. For a given graphical sequence $\sigma$, let $D$ be a digraph of $\sigma$ having the smallest number of strong components. Then either there are lines from every point of $S$ to every point of $S^{\prime}$, or lines from every point of $S^{\prime \prime}$ to every point of $S$.

Proof. Suppose the lemma is false. Since $S$ and $S^{\prime}$ are distinct strong components, there must by [5] be no lines in one direction, say from $S^{\prime}$ to $S$. Then by the assumption, there are points $u$ in $S$ and $v^{\prime}$ in $S^{\prime}$ such that there is no line from $u$ to $v^{\prime}$. Since $S$ and $S^{\prime}$ are nontrivial, they contain lines $x=u v$ and $x^{\prime}=u^{\prime} v^{\prime}$ respectively. Define the two new lines $y_{1}=u v^{\prime}$ and $y_{2}=u^{\prime} v$. Then of course $E=D-x-x^{\prime}+y_{1}+y_{2}$ is also a digraph of $\sigma$. We will show that $E$ has fewer strong components than $D$. Let $w$ and $w^{\prime}$ be points of $S$ and $S^{\prime}$, respectively. In $S-x$, there is a path $P$ from $w$ to $u$ and a path $Q$ from $v$ to $w$; similarly, in $S^{\prime}-x^{\prime}$, there are paths $P^{\prime}$ and $Q^{\prime}$ from $w^{\prime}$ to $u^{\prime}$ and from $v^{\prime}$ to $w^{\prime}$. Therefore

$$
P+y_{1}+Q^{\prime}+P^{\prime}+y_{2}+Q
$$

contains paths from $w$ to $w^{\prime}$ and from $w^{\prime}$ to $w$. Hence, every pair of points are mutually reachable in $E$. Therefore $E$ has fewer strong components than $D$, which is a contradiction, proving the lemma.

Theorem 3. Let $\sigma=\left(\left(a_{1}, b_{1}\right),\left(a_{2}, b_{2}\right), \ldots,\left(a_{p}, b_{p}\right)\right)$ be a graphical sequence in which $a_{1} \geqslant a_{2} \geqslant \ldots \geqslant a_{p}$. Then $\sigma$ belongs to a strong digraph 
if and only if each $a_{i}>0$, each $b_{j}>0$, and for each $k: 1 \leqslant k \leqslant p-1$, the following strict inequalities hold:

$$
\sum_{i=1}^{k} a_{i}<\sum_{i=1}^{k} b_{i}+\sum_{i=k+1}^{p} \min \left\{k, b_{i}\right\}
$$

Proof. To prove the necessity, consider a strong digraph $D$ whose pcints $v_{i}$ are labelled so that $a_{1} \geqslant a_{2} \geqslant \ldots \geqslant a_{p}$. Suppose that for some integer $k$, equality holds in (4). Let $U=\left\{v_{1}, v_{2}, \ldots, v_{k}\right\}$ and let $W=\left\{v_{k+1}, \ldots, v_{p}\right\}$. Any point $v_{i}$ in $W$ can have at most $\min \left\{k, b_{i}\right\}$ lines to it from points of $U$, so there are at most $\sum_{k+1}^{p} \min \left\{k, b_{i}\right\}$ lines from points of $U$ to points of $W$. Because there are $\Sigma_{1}^{k} a_{i}$ lines from points of $U$, there must thus be exactly $\Sigma_{1}^{k} b_{i}$ lines joining points of $U$. From this it follows that no point of $W$ can reach any point of $U$, contradicting the assumption that $D$ is strong.

The proof of the sufficiency of these conditions is considerably longer. We remark that in this proof, whenever a point is labelled with a subscript, e.g., $u_{i}$ or $w_{j}$, that label denotes that point throughout. On the other hand, the meaning of a point $v$ without a subscript may vary as we prove various assertions. The same applies to lines $x_{i}$ with subscripts and lines $x$ and $x^{\prime}$ without. Lines $y_{i}$ may vary during the proof, but these always denote lines not in the given digraph $D$.

Now let us assume that $\sigma$ is a graphical sequence satisfying the stated conditions but not belonging to any strong digraph. Let $D$ be a digraph of $\sigma$ with the minimum number of strong components. There is a strong component $S_{0}$ such that no point of $S_{0}$ is adjacent from any point not in $S_{0}$ by [5]. Since by hypothesis no $b_{i}=0, S_{0}$ must be nontrivial and hence by the lemma is unique. Let $V_{0}$ be the set of points of $S_{0}$, and say it has $m$ points.

Assume there is a point $w_{1}$ not in $V_{0}$ which is not adjacent from $\min \left\{m, \mathrm{id} w_{1}\right\}$ points of $V_{0}$. Let $u_{0}$ and $w_{0}$ be points of $V_{0}$ such that $u_{0}$ is not adjacent to $w_{1}$ but is adjacent to $w_{0}$. Then by the lemma, $w_{1}$ must be a trivial strong component.

Assertion 1. The point $w_{1}$ is not adjacent from any point in any nontrivial strong component other than $S_{0}$. Suppose that this is not the case for some point $v$ in another nontrivial strong component $S$. Let $x_{0}=u_{0} w_{0}$ and $x=v w_{1}$, and define two new lines $y_{1}=u_{0} w_{1}$ and $y_{2}=v w_{0}$. Then $E=D-x_{0}-x+y_{1}+y_{2}$ is a digraph of $\sigma$ with fewer strong components than $D$, because in $E$ all points of $S_{0}$ and $S$ are mutually reachable. This contradiction proves Assertion 1.

From this it follows that there is a point $u_{1}$ of a trivial strong component which is adjacent to $w_{1}$. Without loss of generality, we can take $u_{1}$ as adjacent from $\min \left\{m\right.$, id $\left.u_{1}\right\}$ points of $V_{0}$. For otherwise the same argument as above would apply to $u_{1}$ as it did to $w_{1}$, and after a finite number of steps we would reach points having the properties of $u_{1}$ and $w_{1}$. 
Assertion 2. The point $u_{1}$ is adjacent only from points of $V_{0}$. Suppose it is adjacent from some other point. Then it is adjacent from every point of $V_{0}$. Let $x_{0}=u_{0} w_{0}, x_{1}=u_{1} w_{1}, y_{1}=u_{0} w_{1}$, and $y_{2}=u_{1} w_{0}$. Then $E=D-x_{0}-x_{1}+y_{1}+y_{2}$ has fewer strong components than $D$ since all points of $V_{0} \cup\left\{u_{1}\right\}$ are mutually reachable. Therefore, $u_{1}$ must be adjacent from id $u_{1}$ points of $V_{0}$, and hence is adjacent only from those points.

Assertion 3. The point $u_{1}$ is adjacent to every point of every nontrivial strong component other than $S_{0}$. Suppose $v$ is a point in another nontrivial strong component $S$ and is not adjacent from $u_{1}$. Let $x_{1}=u_{1} w_{1}$, $x=u_{0} v, y_{1}=u_{0} w_{1}$, and $y_{2}=u_{1} v$. Then $E=D-x_{1}-x+y_{1}+y_{2}$ has the same strong components as $D$. But $S_{0}$ and $S$ are nontrivial strong components of $E$, neither of which has every point adjacent from every point of the other. Thus, $E$ satisfies the hypothesis but not the conclusion of the lemma, which proves this assertion.

Let $V_{1}=V_{0} \cup\left\{u_{1}\right\}$. Assume there is a point $w_{2}$ not in $V_{1}$ which is not adjacent from $\min \left\{m+1\right.$, id $\left.w_{2}\right\}$ points of $V_{1}$. By the lemma and Assertion $3, w_{2}$ must be a trivial strong component.

Assertion 4. The point $w_{2}$ is not adjacent from any point in any nontrivial strong component other than $S_{0}$. Again we suppose this is not true. Let $v$ be a point in a nontrivial strong component $S$ and adjacent to $w_{2}$. Then by the same argument as that used in Assertion 1, it follows that $w_{2}$ is adjacent from every point of $S_{0}$. Hence it is not adjacent from $u_{1}$. Let $x_{0}=u_{0} w_{0}, x_{1}=u_{1} w_{1}, x=v w_{2}, y_{1}=u_{0} w_{1}, y_{2}=u_{1} w_{2}$, and $y_{3}=v w_{0}$. Then $E=D-x_{0}-x_{1}-x+y_{1}+y_{2}+y_{3}$ has fewer strong components than $D$, since points of $S_{0}$ and $S$ are now mutually reachable.

Therefore, there is a point $u_{2}$ of a trivial strong component which is adjacent to $w_{2}$, and without losing generality we can consider $u_{2}$ as being adjacent from $\min \left\{m+1\right.$, id $\left.u_{2}\right\}$ points of $V_{1}$.

Assertion 5. The point $u_{2}$ is adjacent only from points of $V_{1}$. Suppose this is not the case. Then $u_{2}$ must be adjacent from every point of $V_{1}$. Also, by the argument used in proving Assertion 2, $w_{2}$ must be adjacent from every point of $S_{0}$. Hence, the line $u_{1} w_{2}$ does not exist in $D$, and since $u_{2}$ is adjacent from every point of $S_{0}, u_{2} \neq w_{1}$. Let $x_{0}=u_{0} w_{0}$, $x_{1}=u_{1} w_{1}, x_{2}=u_{2} w_{2}, y_{1}=u_{0} w_{1}, y_{2}=u_{1} w_{2}$, and $y_{3}=u_{2} w_{0}$. Then the digraph $E=D-x_{0}-x_{1}-x_{2}+y_{1}+y_{2}+y_{3}$ has fewer strong components than $D$, which is impossible.

Assertion 6. The point $u_{2}$ is adjacent to every point of every nontrivial strong component other than $S_{0}$. Once more we suppose the contrary and let $v$ in $S$ be some such point not adjacent from $u_{2}$. By the argument used in Assertion 3, $w_{2}$ is then adjacent from every point of $S_{0}$. Hence the line $u_{1} w_{2}$ does not exist. Let $x=u_{0} v, x_{1}=u_{1} w_{1}, x_{2}=u_{2} w_{2}, y_{1}=u_{0} w_{1}$, $y_{2}=u_{1} w_{2}$, and $y_{3}=u_{2} v$. Then in $E=D-x-x_{1}-x_{2}+y_{1}+y_{2}+y_{3}$, which 
has the same strong components as $D$, there is a point $v$ in a nontrivial strong component which is not adjacent from every point of $S_{0}$. This is a contradiction to the lemma and proves the assertion.

Let $V_{2}=V_{1} \cup\left\{u_{2}\right\}$. If there is a point $w_{3}$ not in $V_{2}$ which is not adjacent from $\min \left\{m+2, \mathrm{id} w_{3}\right\}$ points of $V_{2}$, we repeat this process and get a point $u_{3}$, analogous to points $u_{1}$ and $u_{2}$. After a finite number of repetitions, we obtain a set of points $V_{n}=V_{0} \cup\left\{u_{1}, u_{2}, \ldots, u_{n}\right\}$ such that (i) every point in every nontrivial strong component other than $S_{0}$ is adjacent from every point of $V_{n}$, (ii) every point $v$ of every trivial strong component is adjacent from $\min \{m+n$, id $v\}$ points of $V_{n}$, and (iii) no point of $V_{n}$ is adjacent from any point not in $V_{n}$.

Let $u$ be a point in $V_{n}$ and $w$ a point not in $V_{n}$. It follows that if $w$ is adjacent to a point, then $u$ is also adjacent to that point; hence, $\operatorname{od} u \geqslant \operatorname{od} v$. If $u$ is in $S_{0}$, then it is adjacent to some other point of $S_{0}$, which is not adjacent from $w$. If $u$ is not in $S_{0}$, it is adjacent to some point $v$ not adjacent from every point of $V_{n}$ and hence not adjacent from $w$. Therefore, $\operatorname{od} u>\operatorname{od} w$. Let $k=m+n$. Then

$$
\sum_{i=1}^{k} a_{i}=\sum_{i=1}^{k} b_{i}+\sum_{i=k+1}^{p} \min \left\{k, b_{i}\right\}
$$

This equality establishes the theorem.

\section{Degree sequences of functional digraphs}

A functional digraph is one in which every point has outdegree 1. Putting it ancther way, a functional digraph portrays an irreflexive function from a finite set $V$ into itself. Clearly, in the degree sequence of a functional digraph, each $a_{i}=1$. In the next theorem, we provide a criterion for a sequence of ordered pairs to belong to a functional digraph. We call such a sequence functional.

Theorem 4. Let $\sigma=\left(\left(a_{1}, b_{1}\right),\left(a_{2}, b_{2}\right), \ldots,\left(a_{p}, b_{p}\right)\right)$ be a sequence of ordered pairs of integers in which each $a_{i}=1$ and $0 \leqslant b_{i} \leqslant p-1$. This sequence belongs to some functional digraph if and only if

$$
\sum_{i=1}^{p} b_{i}=p
$$

Proof. The necessity of this equality follows since $\Sigma_{1}^{p} b_{i}=\Sigma_{1}^{p} a_{i}=p$.

For the sufficiency, we show that if $\sigma$ is a sequence in which every $a_{i}=1, b_{i} \leqslant p-1$, and $\sum_{1}^{p} b_{i}=p$, then $\sigma$ is graphical. That it then belongs to a functional digraph is apparent.

It is obvious that (1) of Theorem 0 holds for $\sigma$. Also, at least two of the numbers $b_{i}$ are non-zero. Let $A_{k}, 1 \leqslant k \leqslant p-1$, denote the following sum :

$$
A_{k}=\sum_{i=1}^{k} \min \left\{b_{i}, k-1\right\}+\sum_{i=k+1}^{p} \min \left\{b_{i}, k\right\}
$$


We consider several cases. If $k=1$, there is an $i>1$ for which $b_{i} \geqslant 1$, $A_{1} \geqslant 1$. Now take $k>1$. If every $b_{i} \leqslant k-1$, then $A_{k}=\Sigma_{1}^{p} b_{i}=p>k$. The only remaining case is that in which some $b_{j} \geqslant k>1$. This $b_{j}$ then contributes at least $k-1$ to $A_{k}$ and the other non-zero $b_{i}$ contributes at least 1. Hence in this case as well, $A_{k} \geqslant k$. Therefore, for each $k$, $\sum_{1}^{k} a_{i}=k \leqslant A_{k}$, proving that (2) of Theorem 0 holds for $\sigma$. Hence $\sigma$ is graphical, which serves to establish the theorem.

Strong and weak functional digraphs are characterised structurally in Harary [4]. Using these results, we find criteria for a functional sequence to belong to strong, weak, and disconnected digraphs.

Corollary 4a. Let $\sigma=\left(\left(a_{1}, b_{1}\right),\left(a_{2}, b_{2}\right), \ldots,\left(a_{p}, b_{p}\right)\right)$ be a. functional sequence.

1. $\sigma$ belongs to some strong digraph if and only if each $b_{i}=1$.

2. $\sigma$ belongs to some weak digraph which is not strong if and only if some $b_{i}=0$.

3. $\sigma$ belongs to some disconnected digraph if and only if at least four $b_{i}$ are non-zero.

\section{Degree sequences of traversable digraphs}

An isograph is a digraph in which each point has equal outdegree and indegree. A weak isograph possesses the following interesting property : it has a sequence $u_{1}, u_{1} u_{2}, u_{2}, u_{2} u_{3}, \ldots$ in which points and lines alternate which begins at any point and proceeds in such a way that no line is repeated, that traverses each line exactly once and returns to the starting point. Such digraphs are called traversable, and weak isographs are the only traversable digraphs by a well-known result of Euler.

Our next result provides a criterion for a sequence of ordered pairs to belong to some isograph. We remark that every weak isograph is necessarily strong, see [5]. Therefore, using Theorem 2, a graphical sequence of ordered pairs of equal integers belongs to some strong digraph if and only if it contains no $(0,0)$.

TheOREM 5. Let $\sigma=\left(\left(a_{1}, b_{1}\right),\left(a_{2}, b_{2}\right), \ldots,\left(a_{p}, b_{p}\right)\right)$ be a sequence of ordered pairs of integers in which $0<a_{i}=b_{i} \leqslant p-1$ and $a_{1} \geqslant a_{2} \geqslant \ldots \geqslant a_{p}$. Then $\sigma$ belongs to some traversable digraph if and only if the following $p-1$ inequalities hold:

$$
\sum_{i=1}^{k} a_{i} \leqslant k(k-1)+\sum_{i=k+1}^{p} \min \left\{k, a_{i}\right\} ; \quad 1 \leqslant k \leqslant p-1 .
$$

Proof. If $\sigma$ is a sequence belonging to a traversable digraph, the inequalities (2) of Theorem 0 , and therefore the inequalities (5), hold.

For the converse, we suppose that $\sigma$ satisfies the above conditions, but 
that for some integer $k$,

$$
\sum_{i=1}^{k} a_{i}>\sum_{i=1}^{k} \min \left\{k-1, b_{i}\right\}+\sum_{i=k+1}^{p} \min \left\{k, b_{i}\right\} .
$$

It is obvious that $k \neq 1$. If $a_{k} \geqslant k-1$, then

$$
\sum_{i=1}^{k} a_{i}>\sum_{i=1}^{k} \min \left\{k-1, a_{i}\right\}+\sum_{i=k+1}^{p} \min \left\{k, b_{i}\right\}=k(k-1)+\sum_{i=k+1}^{p} \min \left\{k, b_{i}\right\} .
$$

Because this contradicts the hypothesis that (5) holds for each $k$, it follows that $a_{k}<k-1$. Clearly $a_{1}>k-1$, for otherwise,

$$
\sum_{i=1}^{k} a_{i}=\sum_{i=1}^{k} \min \left\{k-1, a_{i}\right\} \text {, contradicting the supposition. }
$$

Let $j$ be the greatest integer such that $a_{j} \geqslant k-1$. Then

$$
\sum_{i=1}^{k} a_{i}>\sum_{i=1}^{k} \min \left\{a_{i}, k-1\right\}+\sum_{i=k+1}^{p} \min \left\{a_{i}, k\right\}=j(k-1)+\sum_{i=j+1}^{k} a_{i}+\sum_{i=k+1}^{p} a_{i} .
$$

Therefore,

$$
\sum_{i=1}^{j} a_{i}>j(k-1)+\sum_{i=k+1}^{p} a_{i}
$$

By hypothesis,

$$
\sum_{i=1}^{j} a_{i} \leqslant j(j-1)+\sum_{i=j+1}^{p} \min \left\{a_{i}, j\right\} \leqslant j(j-1)+\sum_{i=j+1}^{k} \min \left\{a_{i}, j\right\}+\sum_{i=k+1}^{p} a_{i} .
$$

Combining these two inequalities, we have

$$
j(k-1)+\sum_{i=k+1}^{p} a_{i}<\sum_{i=1}^{j} a_{i}<\sum_{i=1}^{p} a_{i} \leqslant j(j-1)+\sum_{i=j+1}^{k} \min \left\{a_{i}, j\right\}+\sum_{i=k+1}^{p} a_{i} .
$$

Therefore,

$$
j(k-j)<\sum_{i=j+1}^{k} \min \left\{a_{i}, j\right) \leqslant j(k-j) .
$$

This contradiction establishes the theorem since it follows from our earlier remarks that being graphical, $\sigma$ must belong to a traversable digraph.

It is interesting to note the similarity of (5) to the criteria of Erdös and Gallai [3] for a sequence of positive integers $\rho=\left(a_{1}, a_{2}, \ldots, a_{p}\right)$ : $p-1 \geqslant a_{1} \geqslant a_{2} \geqslant \ldots \geqslant a_{p}$ to be the sequence of degrees of some graph (undirected). The only requirement in addition to (5) is that $\sum_{1}^{p} a_{i}$ be even. From this it follows, of course, that the sequence $\sigma$ of Theorem 5 belongs to a symmetric digraph if and only if $\Sigma_{1}^{p} a_{i}$ is even.

\section{Summary}

In this note we have provided criteria for determining whether a sequence of ordered pairs of integers belongs to digraphs of various classes, $v i z$. strong, weak, disconnected, functional, and traversable digraphs. 


\section{References}

1. D. Cartwright and F. Harary, "The number of lines in a digraph of each connectedness category", S.I.A.M. Review, 3 (1961), 309-314.

2. D. R. Fulkerson, "Zero-one matrices with zero trace", Pacific J. of Math., 10 (1960). $831-836$.

3. P. Erdős and T. Gallai, "Graphen mit Punkten vorschriebenen Grades ", Mat. Lapok, 11 (1960), 264-274.

4. F. Harary, "The number of functional digraphs", Math. Annalen, 138 (1959), 203-210,

5. F. Harary, R. Z. Norman and D. Cartwright, Structural models: an introduction to the theory of directed graphs (Now York, 1965).

6. H. Ryser, "Matrices of zeros and ones ", Bull. American Math. Soc., 66 (1960), $442-464$.

The University of Michigan.

University College, London. 